\title{
The Influencing Factor Analysis and Suggestions about College Students' Devotion to Study
}

\author{
Feifei Sun ${ }^{1}$ and Yuanyuan $\mathrm{Cai}^{2}$
}

${ }^{1}$ College of Mechanical and Electrical Engineering, Zaozhuang University Shandong province, 277160;

${ }^{2}$ Zaozhuang Experimental School, Shandong Zaozhaung 277100

\begin{abstract}
With the development of international economy forms, the cultivation of high-quality talents has become one of the most important tasks all over the world. College students shoulder the heavy burden of the development of country and society. How to cultivate college students with high-level science knowledge and skills has become an important task of Chinese higher education teaching. Study is still college students' top task. Positive learning investment is the necessary way for college students to master knowledge and skills. Considering the present situation where college students have not enough devotion to study, this paper analyzes the causes of college students' less devotion to study from the aspects of time management and distribution, psychological energy, and action force. Suggestions about improving the devotion to study are put forward from the aspects of educators' correct realization and guidance, college students' own initiative and willpower improvement.
\end{abstract}

Keywords: College student; Learning inputs; Time management; Mental energy force; Action

\section{大学生学习投入的影响因素分析及其建议}

\author{
孙斐斐 ${ }^{1}$, 蔡媛媛 ${ }^{2}$
}

（1.臬庄学院、机电工程学院，山东 本庄 277160；2．本庄市实验学校，山东 本庄 277100 ）

摘要: 随着国际经济形式的发展, 高素质人才的培养已成为世界各国的重要任务之一。大学生肩负着国家和社会发展的 重担, 如何培育掌握高水平科学知识技能的大学生已成为我国高等教育教学的重要任务。学习仍是大学生的首要任务, 积极 的学习投入是大学生掌握知识技能的必经之路。鉴于当下大学生学习投入不足的现状, 本文从时间管理和分配、心理能量、 行动力三个方面分析了大学生学习投入不足的原因, 并从教育者的正确认识、教育者的正确引导、大学生自身的主动性和意 志力提高三个方面提出了促进大学生学习投入的建议。

关键词: 大学生; 学习投入; 时间管理; 心理能量; 行动力

中图分类号: B844 文献标志码: A

引言

在经济全球化发展的时代背景下, 高素质人才的培养成为每个国家高等教育面临的一个共同话题。如 何提高我国高等教育的教学质量? 从高等教育的主体一大学生的角度来讲, 积极的学习投入无疑是衡量 高等教育质量的一个重要指标。同时, 大学生积极的学习投入也是我国高素质人才培养的必然途径。所谓 外因通过内因起作用, 如果高等教育无法引导大学生更多积极的学习投入, 那再多优惠的教育政策、良好 的教育环境都只是摆设。所以, 探讨影响大学生学习投入的因素, 从而探索促进大学生学习投入的方法有 着重要的社会现实意义。

20 世纪 30 年代, 泰勒首次提出学习投入概念, 20 世纪 80 年代学习投入成为国外教育界关注的研究 对象之一。乔治库认为, 学习投入是指学生在有效教育活动上投入的时间和精力 [1]。弗雷德里克斯从认 
知、情感和行为三个维度来建构学习投入。综合前人对学习投入的界定, 本文认为学习投入是指学生在所 有学习活动中投入的全部时间、心理能量和行动力。

\section{1 大学生学习投入的现状描述}

从大学生学习投入的时间内容上看, 大学生对学习的时间投入主要分配在专业课学习上, 也就是说大 学生将大部分学习时间用来学习专业课。大学生对时间的管理和控制能力较差, 一方面表现在大学生在空 闲时间往往不会选择用来学习, 或者到自习室、图书馆后打开书本却聊天或看手机, 做与学习无关的事情, 学习效率较低 $[2]$ 。

本文将大学生学习投入的心理能量分为认知和情感两个成分。从大学生对学习投入的认知方面来看, 大学生对学习策略的认知水平较低, 谢睿在大学生专业课学习投入及其对学习结果的影响研究中发现, 过 半的大学生采用的还是重复记忆、依赖老师等较低级的学习策略。在与大学生的访谈中, 本文也发现了与 上述研究同样的结论。从大学生对学习投入的情感方面来看, 大学生对学习投入的情感水平较高, 从与大 学生的访谈中, 本文发现, 90\%以上的大学生都希望自己好好学习, 并认为能从学习的成功中获得成就感 和愉快的情绪体验。但是, 情绪往往是伴随着行为产生的, 由于大学生的学习行为执行力较差, 所以期望 的学业成功往往不能实现, 从而产生焦虑、消极的情绪体验, 这种在情绪期望与情绪体验之间的巨大反差, 往往会严重打击大学生的学习投入热情。

从大学生学习投入的行为方面来看, 大学生的学习行为普遍表现出行动力差的特点。“理想丰满, 现 实骨感” 的现状普遍存在于大学生的学习行为中。想的多说的多而做的少, 甚至不去做的现象在大学生身 上已成为一种常态。虽然, 大部分大学生对自己没有履行自我学习承诺存在内疚、焦虑等消极情绪, 但仍 然没有避免这种现象的发生。

\section{2 影响大学生学习投入的因素分析}

\section{1 影响大学生学习投入的时间因素分析}

学习投入需要时间做保障。通过阅读大量文献，本文发现，时间作为学术研究中的一个变量主要从两 个方面来考虑，一方面是时间分配，另一方面是时间管理和时间控制。

对大学生时间分配进行的研究非常少，其中对时间分配做较详细研究的是刘佳丽所做的基于心理账户 理论的大学生时间分配研究。研究发现, 过半数的大学生都有时间分配的意识, 但总体对时间分配的概念 较模糊。同时, 现代大学生分配在娱乐活动上的时间要远远大于分配在学习上的时间, 也就是说他们更愿 意将时间分配在娱乐活动中。这种现象传递出来的信息是大学生对学习的主动性较差, 或者说从大学生的 学习行为上来看, 大学生似乎不愿意花时间用来学习。

对大学生的时间管理和时间控制方面的研究比较丰富, 但很少将其作为学习投入的一个变量来研究。 本文在与学生的大量访谈中发现, 相当一部分大学生在学习之余不知道该做什么, 即便将空余时间用来进 行课程的复习和讨论等学习活动, 其效率往往也很低, 并且学习内容在时间上没有连贯性, 不同时间段的 学习内容往往不同, 想到哪儿学哪儿, 想到什么学什么。在时间管理的系统性上表现较差, 或者说时间管 理的能力较差 [5]。这种现象传递出来的信息是大学生不知道如何系统的安排时间, 高效的使用时间。

大学生在时间分配和时间管理方面规划不理想的前提下，很难谈能有较高的学习投入。用在学习上的 时间不足, 学习投入将无法开展; 不能合理的管理和安排时间, 学习投入的效果必然不理想。造成这一困 
境的原因主要是我国的教育工作者和家长过于投入甚至干涉孩子的学习活动, 从小学到中学, 一直沿袭着 学生在教师的督促下学习, 孩子在家长的催促中努力, 在学生的学习活动中, 教师和家长的过度参与无形 中剥夺了学习活动主体一学生在时间方面的自我管理。到了大学, 时间宽裕了, 教师和家长对学习活动的 干预骤然下降, 学生不会管理和分配时间的弱点就被彰显的更加明显, 以至影响到在大学阶段的学习投入。

\section{2 影响大学生学习投入的心理能量因素分析}

本文从心理能量的两个构成成分认知和情感来分析对学习投入的影响。首先, 周开发在对地方院校工 科生学习策略、问题解决能力与学业成绩的相关性研究中发现, 大学生的学习策略水平总体偏低。张伟新 在对护理专业大学生自主学习能力和学习策略现状及相关性分析中发现, 护理专业大学生的学习策略总体 水平中等。本文认为护理专业女生更多, 在以上研究中也都发现, 女生的学习策略一般要好于男生, 因此, 得到总体水平中等的结论。本文结合对大学生的访谈得出与前人研究一样的结论, 大学生的认知学习策略 层次较低。大学生使用较多的学习策略是反复记忆、上课认真听讲, 按时完成老师布置的作业, 而深层次 分析学习内容, 扩充学习领域, 主动与老师同学深入探讨问题等学习策略使用较少。也就是说, 大学生的 学习策略还是延续高中时的方式, 没有随着大学学习特点的改变而改变。这种现象的发生也与现有教育体 制下教育者和家长的教育方式存在很大关系。低层次的学习策略导致较低的学习效率, 在付出时间和精力 却得不到高效的学习效果的情况下, 大学生的自我效能感必然会下降, 从而进一步动摇学习投入的主动性 和坚持性, 导致不愿意进行学习投入或者学习投入效率低。其次, 在情感方面, 大部分大学生对学习投入 的情感都较积极, 表示愿意付出时间和精力进行学习, 但由于学习投入的低效率和学习投入过程中遇到的 困难, 以及学习投入的高情感期待与低行动执行之间的反差导致愧疚和焦虑 [8], 从而影响大学生进一步 克服困难进行学习投入。情绪是伴随着行为产生的, 同时又影响着行为的发生。相当一部分大学生的学习 投入都处在高情感期待，低行动执行，高愧疚高焦虑的状态中，无法突破这个恶性循环。

\section{3 影响大学生学习投入的行动力因素分析}

行动是学习投入发生的前提和充分条件, 行动力差是大学生学习投入差的主要原因, 更是大学生不愿 面对却又必然存在的障碍。通过访谈发现, 绝大部分大学生都是有理想有抱负的, 在理想和抱负陈述完毕 之后便是 “但是” , 但是之后的内容无外乎三点, 一是不行动的理由和借口, 二是不行动伴随着的负面情 绪体验, 三是自我反思后的下一次行动承诺。这种现象传递出的信息是相当一部分大学生是思想的巨人, 行动的矮子。没有行动力的发生, 学习投入就无从谈起 [9]。

\section{3 促进大学生学习投入的建议}

\section{1 高校教育者要从深层次理解大学生学习投入不足的原因}

人的发展是一个连续的过程, 大学生在学习投入方面表现出来的特点是其整个学习生涯中教育因素的 必然产物。我国现行的教育体制实质仍具有重知识重升学的特点, 从小学到中学, 教师对学生在学习方面 的严格要求要远远大于学生本人对自己的要求, 同样, 家长对孩子在学习方面的期望和要求也远远大于孩 子本人。似乎学习不再是学生的事情, 而是老师、班主任、家长这个庞大教育者和监护者群体的事情, 学 生本身却成了学习的工具。学习主体地位的颠倒导致学生学习主动性的缺失。小学和中学有教师和家长的 督促和安排, 大学给了学生更多的时间, 需要大学生自己安排和自我监督, 而这些在大学生学业生涯中的 经验微乎其微。作为高校教育者从教育和人的心理发展的角度应该更能理解大学生呈现出来的学习投入不 
足的现象, 应该及时给予正确的引导, 而不是一味的批评指责大学生不爱学习, 不好好学习, 不愿意学习 的行为。

\section{2 高校教育者要正确引导大学生进行积极的学习投入}

大学生对学习投入的情感期望较高, 由此可以看出, 大学生从情感上是愿意学习, 愿意进行学习投入 的。但是由于大学生在学习投入方面的经验较少, 行动力较差, 意志力较薄弱, 如何引导大学生进行积极 的学习投入将成为高校教育者的重要任务。本文认为, 高校教育者应从以下三个方面引导大学生进行积极 的学习投入。

首先, 要让大学生从思想上明确, 行动是一切理想得意实现的唯一途径。如果没有学习行动力, 对学 习投入的情感再高都只是想象。其次, 在学习策略上要给予大学生一定的指导。大学不同于高中, 大学不 再是老师说什么就要做什么的场所, 而应该是在老师的引导和帮助下做与增强自己专业技能, 涉足自己感 兴趣的学科领域, 对自己发展有意义的事情的场所。应该改变死记硬背、仅仅依赖老师学习课本知识的低 层次学习策略。高校教育者在教育教学中应该启发学生提出问题、指导学生解决问题, 与学生讨论, 引导 学生查阅资料, 培养学生的专业兴趣, 深入思考, 从而培养大学生较高层次的学习策略 [10]。再次, 帮助 大学生合理的规划时间, 进行科学的时间管理和有效的时间分配。大学生积极的学习投入能够帮助大学生 实现专业领域知识技能的高效学习和拓展感兴趣领域的知识。同时，大学生积极的学习投入又能够帮助大 学生节省更多的时间用于兼职或做其他事情, 促进大学生的全面发展, 增加就业能力。

\section{3 大学生应增强学习投入的主动性和意志力}

所谓 “十年寒窗无人问”, 学习本身是一件辛苦的事情, 大学生经历了近十年的学习, 到了大学突然 有了一种苦尽甘来的轻松感, 学习这件苦差事似乎可以暂时闲置一下, 加上, 大学之前的学习主要是被动 学习, 导致学习投入的主动性较差, 主动努力的学习便成为想象中的事情。如果再加上学习投入过程中遇 到困难, 那学习投入便戛然而止了。所以大学生应该意识到, 大学已将学习的主动权归还自己, 大学生应 该学会自觉高效的组织自己的学习生活。同时, 大学时期是一个人一生中智力发展最好的时期, 学习还是 生活的主旋律, 更应该进行积极主动的学习投入, 为将来的就业和生存储备专业知识和技能。大学生还要 意识到, 学习作为人一生中的一项任务, 遇到困难是必然的, 面对学习投入过程中暂时的情感焦虑, 不能 管理和控制好时间, 行动力不足等困难, 要充分发挥意志力的作用, 积极主动寻找解决困难的办法, 将学 习投入坚持下去。在坚持的过程中也能够进一步增强自身对时间、学习策略、情感和行动力的深入理解和 认识, 进而发生更多更好的学习投入, 形成学习投入过程的良性循环。

\section{4 总结}

大学生是国家和社会健康发展的人才资源, 大学生在知识领域的高水平发展能带动国家和社会经济的 迅速发展。大学生正处在人的智力发展的最顶峰, 努力学习知识技能, 掌握更高的科学文化是其重要的任 务。所以高校教育者要帮助大学生进行积极的学习投入, 把宝贵的时间用在高效学习上, 为将来促进国家 和社会的发展奠定扎实的知识基础。

\section{参考文献:}

[1] 蒋华林, 李华. 学习性投入调查: 本科教育质量保障的新视角 [J]. 高教发展与评估, 2010, vol26：45-47.

[2] 林思涵. 国内外大学生学习投入研究述评 $[J]$. 邵通学院学报, 2015, vo137：111-115.

[3] 谢睿. 大学生专业课学习投入及其对学习结果的影响研究 [D]．2015，46-50; 
[4] 刘佳丽. 基于心理账户理论的大学生时间分配研究[D]. 2014，20-21.

[5] 王春英. 大学生时间管理现状及对策 $[J]$ ，成都航空职业技术学院学报，2016，vo132：25-27.

[6] 周开发. 地方院校工科生学习策略_问题解决能力与学业成绩的相关性研究 [J]. 重庆高教研究, 2016, vol4: 110-116.

[7] 张伟新. 护理专业大学生自主学习能力和学习策略现状及相关性分析 $[J]$. 中国医药导报, 2016, vol13； 77-80.

[8] 杨立军. 大学生学习投入的影响因素及其作用机制 $[J]$. 高教发展与评估，2016，vo132：50-56.

[9] 冯亚乾. 大学学习投入的研究述评 $[J]$. 教育教学论坛, 2015, vo15：76-77.

[10］王威威. 大学生学习策略对教育收获的影响机制分析[D]. 2016, 17-19.

\section{References:}

[1] Jiang Hualin, Li Hua. Investigation of learning inputs: New Perspective of Undergraduate Teaching Quality Guarantee [J].Higher Education Development and Evaluation, 2010, vol26: 45-47.

[2] Lim Sihan. A review on learning engagement of domestic and foreign students [J].Journal of ZhaoTong University,2015, vol37:111-115.

[3] Xie Rui. Research on College students' Professional Learning Inputs and Their Impact on Learning Outcomes [D].2015, 46-50.

[4] liu Jiali. Research on College Students' Time Allocation Based on Mental Accounting Theory [D].2014, 20-21.

[5] Wang Chunying. Present Situation and Countermeasures of College Students' Time Management [J], Journal of Chengdu Aeronautic Vocational and Technical College, 2016, vol32:25-27.

[6] Zhou Kaifa. Learning Strategies of Engineering Students in Local Universities_ Study on Correlation between Academic Achievement and Problem Solving Skills [J].ChongQing Higher Education Research, 2016, vol4: 110-116.

[7] Zhang Weixin. Autonomous Learning Ability and Learning Strategies and Related Analysis on College Students' in Nursing Major [J].China Medical Herald,2016, vol13;77-80.

[8] Yang Lijun. Study on Influence Factors of College Students and its Mechanism [J].Higher Education Development and Evaluation, 2016, vol32: 50-56.

[9] Feng Yaqian. Student Review of Research on University Learning Engagement [J].Education Teaching Forum, 2015, vol5: 76-77.

[10] Wang Weiwei. Analysis of Influence Mechanism of Learning Strategies on Education of College Students to Educational Gains [D].2016, 17-19. 